\title{
A General Discrete Patches Approach to Investigate the Populational Dynamics of Mosquitoes and Humans in Dengue
}

\author{
Fernando Luiz Pio dos Santos ${ }^{1}$ \\ Institute of Biosciences, IB, Botucatu, SP, \\ Department of Biostatistic, Unesp, Botucatu, SP
}

\begin{abstract}
.
In areas where resources are located in patches or discrete locations, human dispersal is more conveniently modeled, in which the population is divided into discrete patches. In this work we develop a general discrete model to analyze the spread of Dengue disease. In the process of mathematical modeling we take into account the human populations and the circulation of a single serotype of dengue mosquitoes. The movements of susceptible, infected and recovered humans between all patches is considered. Aquatic phase with differents carrying capacities is considered within the patches. In this formulation an arbitrary number of patches can be used to simulation of the spread of dengue disease. Numerical results was performed in order to show the applicability of this methodology for the dengue disease problem.
\end{abstract}

Keywords. Human dispersal, discrete model, pathway approach, ODE system.

\section{Introduction}

The simple approach for the analysis of the spread of infectious diseases was initially studied in [1-3]. In this approach, the population in which a pathogenic agent is active can be divided into three distinct compartments corresponding to different epidemiological status: Susceptible $(S)$, Infected $(I)$ and Recovered $(R)$, i.e., the classical SIR model. Removed individuals can be return to the susceptible compartment and then the SIRS is obtained. The model involves the dynamic interactions between humans and mosquitoes and takes into account human mobility as an important factor of disease spread. Humans are the main amplifying host of the virus. Dengue virus circulating in the blood of viraemic humans is ingested by female mosquitoes during feeding. The virus then infects the mosquito midgut and subsequently spreads systemically over a period of 8-12 days. After this extrinsic incubation period, the virus can be transmitted to other humans during subsequent probing or feeding [4]. In the next sections, we describe a discrete model to analize the dynamic of the dengue disease where the movements of humans (susceptible and infected) has a important role when patches are considered.

\footnotetext{
${ }^{1}$ flpio@ibb.unesp.br
} 


\section{Mathematical Epidemic Models}

Due to population dispersal [5] an epidemic model was proposed to describe the dynamics of disease spread among patches. Extending the classical mathematical epidemic model using pathways, we can analyze how different transmission pathways affect the dynamic of the dengue disease. Then, the new methodology $k-A S I R S I$ proposed here consist in extend the classical mathematical model $S I R S I[8,9]$, for an arbitrary number of $k$-patches and also considering a aquatic phase compartment. The state variables definition for the discrete model can be found in Table (1). The respectives parameters are shown in Table (2). Considering all assumptions, the corresponding model is given by the System (1), together $N_{h j}=S_{h j}+I_{h j}+R_{h j}$, for $j=0, \cdots, k$, where $k$ is the number of patches.

Table 1: States variables definition for the discrete model (1).

\begin{tabular}{ll}
\hline$A$ & Aquatic phase \\
$S_{h}$ & Susceptible human density \\
$I_{h}$ & Infected human density \\
$R_{h}$ & Recovered human density \\
$S_{v}$ & Susceptible vector density \\
$I_{v}$ & Infected vector density \\
\hline
\end{tabular}

Table 2: Parameters for the discrete model (1).

\begin{tabular}{ll}
\hline$\mu_{A}$ & per capita aquatic mortality rate \\
$\mu_{h}$ & per capita human mortality rate \\
$\mu_{v}$ & per capita mosquito mortality rate \\
$C$ & carrying capacity related to the amount of available nutrients and space \\
$\phi$ & is the intrinsic oviposition rate \\
$\alpha$ & proportion of mosquito becoming susceptible \\
$b$ & proportion of the effective bite that transmite infection \\
$\gamma$ & recovery rate of humans \\
$\beta_{h}$ & vector-human probability of transmission \\
$\beta_{v}$ & human-vector probability of transmission \\
$N_{h}$ & Total number of humans \\
$K_{i j}$ & humans movement rate between compartment $i$ to $j$ \\
\hline
\end{tabular}




$$
\left\{\begin{aligned}
\frac{d A_{j}}{d t} & =\phi\left(1-\frac{A_{j}}{C_{j}}\right)\left(S_{v j}+I_{v j}\right)-\left(\alpha+\mu_{A}\right) A_{j} \\
\frac{d S_{h j}}{d t} & =\mu_{h}\left(N_{h j}-S_{h j}\right)-\frac{1}{N_{h}} \beta_{h j} b_{j} I_{v j} S_{h j}+\sum_{j=0}^{k} K_{j l} S_{h l}-\sum_{j=0}^{k} K_{l j} S_{h j}, l=0, \cdots, k(l \neq j) \\
\frac{d I_{h j}}{d t} & =\frac{1}{N_{h}} \beta_{h j} b_{j} I_{v j} S_{h j}-\left(\mu_{h}+\gamma\right) I_{h j}+\sum_{j=0}^{k} K_{j l} I_{h l}-\sum_{j=0}^{k} K_{l j} I_{h j}, l=0, \cdots, k(l \neq j) \\
\frac{d R_{h j}}{d t} & =\gamma I_{h j}-\mu_{h} R_{h j}+\sum_{j=0}^{k} K_{j, l} R_{h l}-\sum_{j=0}^{k} K_{l j} R_{h j}, l=0, \cdots, k(l \neq j) \\
\frac{d S_{v j}}{d t} & =\alpha A_{j}-\frac{1}{N_{h}} \beta_{v j} b_{j} I_{h j} S_{v j}-\mu_{v} S_{v j} \\
\frac{d I_{v j}}{d t} & =\frac{1}{N_{h}} \beta_{v j} b_{j} I_{h j} S_{v j}-\mu_{v} I_{v j}
\end{aligned}\right.
$$

\section{Numerical Simulation}

To show the ability of the code in simulate the dynamic of dengue disease involving $k$ discrete patches, we performed simulations for $k=1, k=2$ and $k=5$. The respective matrix human movement rate, $K$, used in the $2-A S I R S I$ and $5-A S I R S I$ models are given by a random process. In the particular case $1-A S I R S I$ model, $K=0$. The ordinary differential equations of the system (1) was discretized by a forward first-order scheme. Higher-order discretization schemes can be considered, but yielding no improvement. The parameters used for all simulations are shown in the Tables (3).

Table 3: Parameters of simulation $\left(\right.$ days $\left.^{-1}\right)$.

\begin{tabular}{cccccccc}
\hline$C$ & $\mu_{A}$ & $\mu_{h}$ & $\mu_{v}$ & $\phi$ & $\alpha$ & $b$ & $\gamma$ \\
\hline $13[7]$ & $0.0583[10]$ & $0.457 \times 10^{-4}[8]$ & $0.25[8]$ & $6.353[10]$ & $0.121[10]$ & $1.0[8]$ & $0.167[8]$ \\
\hline \hline$\beta_{h}$ & $\beta_{v}$ & \multicolumn{10}{c}{} \\
\hline $0.4[8]$ & $0.4[8]$ & & & & \\
\hline
\end{tabular}

Table 4: Initial conditions for the $5-A S I R S I$ model for all patches $j=1, \cdots, k=5$.

\begin{tabular}{llllll}
\hline & 1 & 2 & 3 & 4 & 5 \\
\hline$A$ & 100 & 0 & 0 & 100 & 0.0001 \\
$S_{v}$ & 2000 & 0 & 2000 & 2000 & 0 \\
$I_{v}$ & 0 & 0 & 10 & 0 & 0 \\
$S_{h}$ & 90000 & 100000 & 100000 & 100000 & 100000 \\
$I_{h}$ & 2000 & 0 & 0 & 0 & 0 \\
$R_{h}$ & 0 & 0 & 0 & 0 & 0 \\
\hline
\end{tabular}

The dynamic of $1-A S I R S I$ model show that in the presence of the human infected in the patch, the mosquito susceptible population becomes infected, as shown in Figure (1). 
Also, the low initial density of aquatic phase increase very fast, Figure (2). Figures (3)-(4) show the dynamic of all populations of the $2-A S I R S I$ model. In this dynamic, due to the presence of mosquito infected in the patch 1 , the human susceptible population becomes infected in this patch 1 . Because of the human mobility considered between the patches, the human infected population can move to 2 from 1 and the susceptible mosquitoes in this patch 1 becomes infected. Finally, the human population infected increase in the patch 2 until recover to. In order to show the ability of the code in simulate in $k$ number of discrete patches, we simulated the $5-A S I R S I$ model. Table (4) show the initial conditions for all patches $j=1, \cdots, k=5$. Figures (5) (a) and (b) show the dynamics of humans and mosquito infected populations, respectively. Figure (6) show the dynamic behavior of all populations within all patches. It is important note the effect of human mobility between all patches and their impact in the infection of both susceptible populations.

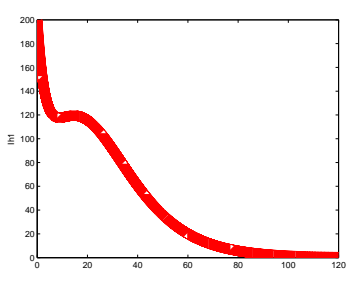

(a) Humans

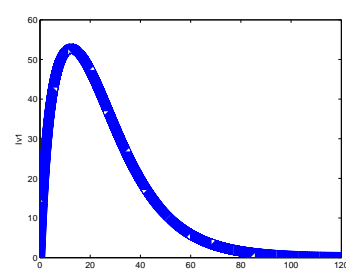

(b) Mosquitoes

Figure 1: Dynamic of the infected populations within the patch in $T=120$ days. Initial conditions: (a) $I_{h 1}(0)=200$; (b) $I_{v 1}(0)=0$.

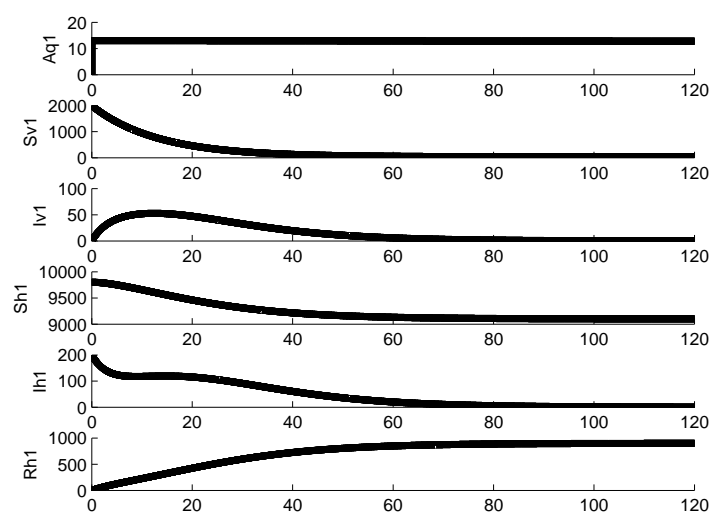

Figure 2: Dynamic population of $1-A S I R S I$ model. Parameters: $A=0.0001 ; N h=$ $10000, I_{h}=200, S_{v}=2000 ; \mathrm{T}=120$ days; $d t=0.01 ; K=0 ; k=1$. 


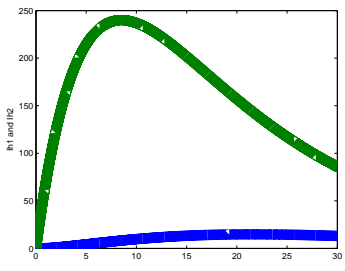

(a) Humans

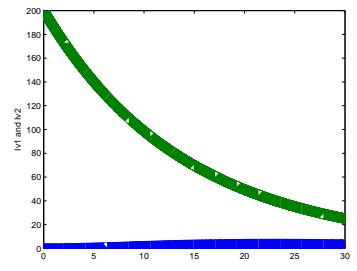

(b) Mosquitoes

Figure 3: Dynamic of the infected populations within the patches. $T=30$ days. Initial conditions: (a) $I_{h 1}(0)=I_{h 2}(0)=0$. (b) $I_{v 1}(0)=200$ and $I_{v 2}(0)=0$.
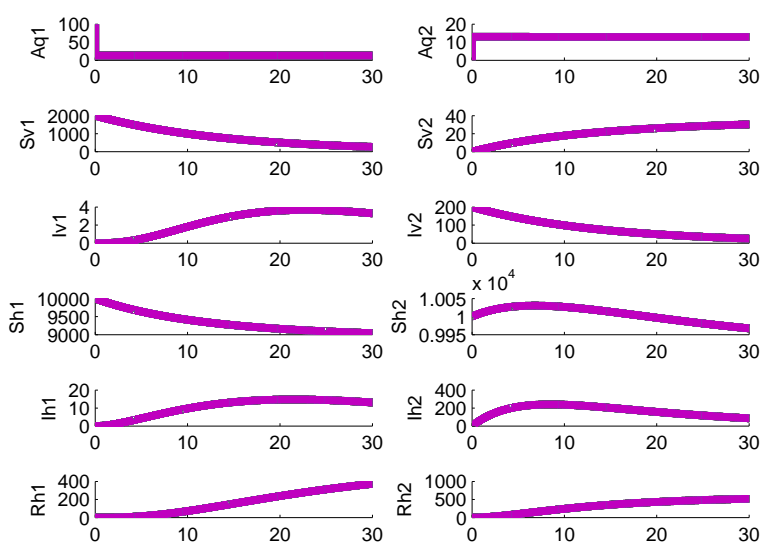

Figure 4: Dengue disease spread using the $2-A S I R S I$ model. Parameters: $A_{1}(0)=100$, $A_{2}(0)=0.0001, S_{v 1}(0)=2000 ; N_{h 1}=N_{h 2}=10000 ;$ Total time T $=30$ days; Time-step $d t=0.01 ; k=2$.

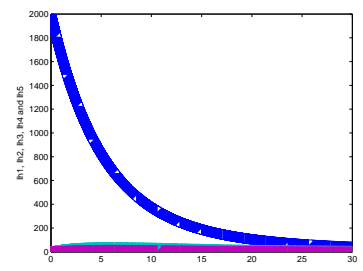

(a) Humans

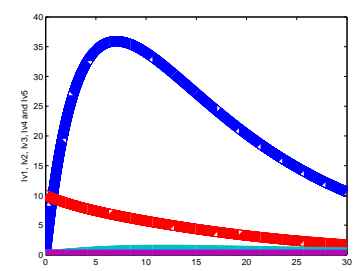

(b) Mosquitoes

Figure 5: Dynamic of the infected populations within the patches. 


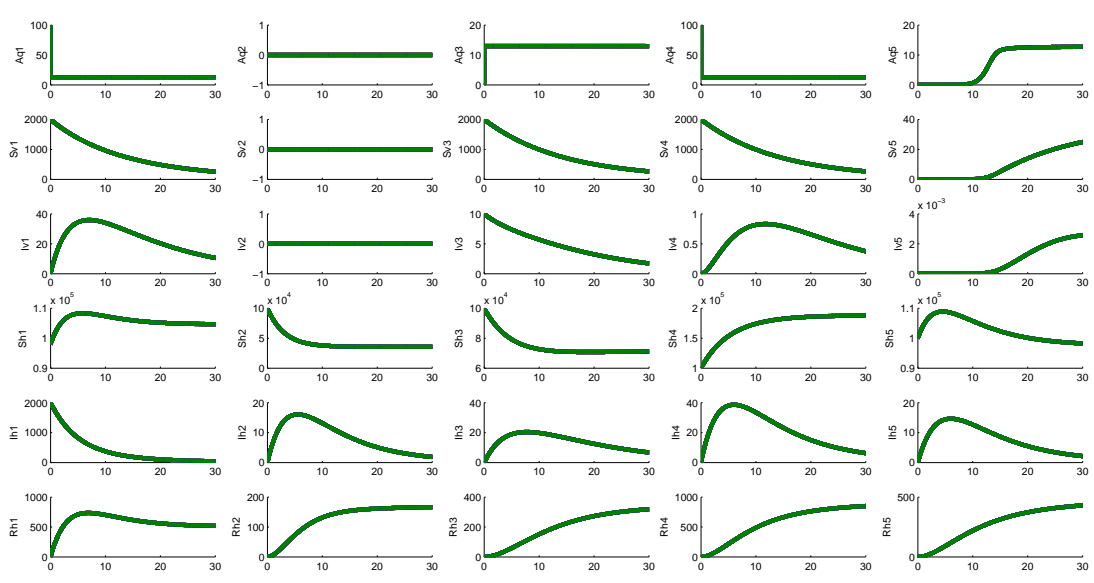

Figure 6: Dengue disease spread using the $5-A S I R S I$ model. Parameters: Initial conditions for all state variables are given in Table (4); Total time: $\mathrm{T}=30$ days; Timestep: $d t=0.01$; Number of patches: $k=5$.

\section{Conclusions}

The main conclusions that can be drawn are:

1. the methodology proposed here allowed the use of an arbitrary number of patches;

2. due to the infected human populations mobility the susceptible mosquito population became infected.

3. the human movement rate is an important parameter and needs to be estimated in order to improve reality in this computational investigation.

\section{Acknowledgments}

The author would like to thank the Professors Claudia P. Ferreira (Institute of Biosciences of Botucatu, IBB/Unesp) and Bedr'Eddine Ainseba (Institute of Mathematical of Bordeaux, IMB) for insightful discussions and suggestions.

\section{References}

[1] W. H. Hamer, Epidemic disease in England. Lancet 1, 733-739, (1906).

[2] R. Ross, The prevention of malaria, 2nd edn. John Murray, London, (1911).

[3] W. O. Kermack, A. G. McKendrick, Contributions to the mathematical theory of epidemics, Part I. Proc R Soc Lond A 115, 700-721, (1927). 
[4] World Health Organization. Dengue: Guidelines for Diagnosis, Treatment, prevention and control, 2nd edn. Geneva: WHO, (2009).

[5] W. Wang, X. Q. Zhao, An epidemic model in a patch environment. Mathematical Biosciences, vol. 190, 97-112, (2004).

[6] E. Kouokam, P. Auger, H. Hbid, M. Tchuente, Effect of the Number of Patches in a Multi-patch SIRS Model with Fast Migration on the Basic Reproduction Rate. Acta Biotheor, vol. 56, 75-86, (2008).

[7] H. O. Florentino, B. F. Bannawart, D. R. Cantane, F. L. P. Santos, Multiobjective genetic algorithm applied to dengue control. Mathematical Biosciences, vol. 256, 1-17, (2014).

[8] H. Nishiura, Mathematical and Statistical Analyses of the Spread of Dengue. Dengue Bulletin, vol. 30, (2006).

[9] M. Andraud, H. Niel, C. Marais, P. Beutels, Dynamic Epidemiological Models for Dengue Transmission: A Systematic Review of Structural Approaches. PLoS ONE 7, vol. 11, (2012).

[10] R. C. A Thomé, H. M. Yang, L. Esteva, Optimal control of Aedes aegypti mosquitoes by the sterile insect technique and insecticide, Mathematical Biosciences, Elsevier, vol. $223,12-23,(2010)$ 